\title{
Generic Eigenvalue Assignment for Generalized Linear First Order Systems using Memoryless Real Output Feedback
}

\author{
Joachim Rosenthal ${ }^{*}$ \\ Department of Mathematics \\ University of Notre Dame \\ Notre Dame, IN 46556-5683 \\ U.S.A. \\ Joachim.Rosenthal@nd.edu \\ Xiaochang Alex Wang ${ }^{\dagger}$ \\ Department of Mathematics \\ Texas Tech University \\ Lubbock, TX 79409-1024 \\ U.S.A. \\ mdxia@ttacs.ttu.edu
}

\author{
J. M. Schumacher \\ CWI, P.O. Box 94079, 1090 GB Amsterdam, \\ and Tilburg University, \\ CentER and Department of Economics, \\ P.O. Box 90153, 5000 LE Tilburg, \\ The Netherlands. \\ Hans.Schumacher@cwi.nl \\ Jan C. Willems \\ Mathematics Institute, \\ University of Groningen, \\ P.O. Box 800, 9700 AV Groningen; \\ The Netherlands. \\ J.C.Willems@math.rug.nl
}

\begin{abstract}
We show in this paper that arbitrary pole assignment by memoryless feedback is generically possible in the class of generalized first order system of a fixed McMillan degree $n$ as soon as $n$ is strictly less than the input number times the output number.

Our result generalizes results by the authors covering the situation when the set of plants consists of strictly proper transfer functions only.
\end{abstract}

\section{Introduction}

The problem of pole assignment by static output feedback has a history going back 25 years. In 1992 Wang [1] proved the surprising result that arbitrary pole placement by static output feedback is generically possible for strictly proper plants having $n$ states, $m$ inputs, and $p$ outputs as soon as $n<m p$. The proof of Wang's result required a considerable amount of techniques from algebraic geometry. More recently Rosenthal, Schumacher and Willems [2] presented a di-

*Supported in part by NSF grant DMS- 9400965 .

${ }^{\dagger}$ Supported in part by NSF grant DMS-9500594. rect and elementary proof of the same result. In this note we show that the same result holds true if the set of plants consists of generalized first order systems having a fixed McMillan degree $n$, a fixed input number $m$ and a fixed output number $p$.

In the sequel we will quickly review the results in $[2,1]$ and related work by Leventides $[3,4]$, Wang [5] and Ariki [6]. In Section 2 we formulate the main results of this paper and in Section 3 we will explain the main ingredients needed in the proof. Our main references for this paper are $[2,7]$.

Let $A, B, C$ be real matrices of size $n \times n, n \times m$ and $p \times n$ respectively. The matrices $A, B, C$ define a linear system with $m$ inputs, $p$ outputs, and $n$ states through:

$$
\frac{d}{d t} x=A x+B u, \quad y=C x .
$$

The problem of generic real eigenvalue assignment by memoryless output feedback seeks for each real monic polynomial $\phi$ of degree $n$, a memoryless real feedback law $u=K y$ such that the controlled system $\frac{d}{d t} x=(A+B K C) x$ has char- 
acteristic polynomial $\phi$ :

$$
\operatorname{det}(s I-A-B K C)=\phi(s) .
$$

To be precise, identify the set of monic real polynomials of degree $n$ with the vector space $\mathbb{R}^{n}$ and identify the set of compensators of size $m \times p$ with the vector space $\mathbb{R}^{m p}$. Then the problem asks for conditions which guarantee that the pole placement map

$$
\begin{aligned}
\chi: \quad \mathbb{R}^{m p} & \longrightarrow \mathbb{R}^{n} \\
K & \longmapsto \operatorname{det}(s I-A-B K C)
\end{aligned}
$$

is surjective. By a simple dimension argument one concludes that $\chi$ cannot be surjective if $n>$ $m p$. The main result by Wang states:

Theorem 1 If $n<m p$ then the pole placement map $\chi$ is surjective for a generic set of real matrices $(A, B, C)$ of sizes $n \times n, n \times m$ and $p \times n$ respectively.

The original proof by Wang [1] was formulated using algebraic geometric methods and the generic set of pole assignable plants was described as a Zariski open subset of a variety parameterizing all strictly proper transfer functions.

Seemingly independently it has been recognized by Leventides [3, 4], Wang [5] and Ariki [6] that geometric techniques used in [1] can be explained to a large part through a linearization of the pole placement map around a so-called 'dependent' compensator. - We will say more about this important technique later in the paper. - Still the proofs in $[4,5,6]$ are all based on polynomial descriptions. In order to use the concept of genericity this requires to equip the set of polynomial matrices of a fixed McMillan degree with the structure of an algebraic variety, which can be done but is a nontrivial task. In Section 4 of this note we will clarify on this point further.

Because of this reason Rosenthal, Schumacher and Willems [2] derived a direct proof which stayed strictly in the state space framework. In this way the translation from the state space framework to the polynomial setting was avoided and the problem was solved in a way it was originally formulated.
In the next section we will show that Theorem 1 is still true if the set of possible plants consists out of generalized first order systems having a fixed McMillan degree, fixed input number and a fixed output number.

\section{Main Result}

Let $K, L$, and $M$ be matrices of sizes $(n+p) \times$ $n,(n+p) \times n$, and $(n+p) \times(m+p)$ respectively. As explained in detail in $[8,9]$ the triple $(K, L, M)$ describes a general linear time-invariant dynamical systems having McMillan degree $n$, input number $m$ and output number $p$ through the equations:

$$
\frac{d}{d t} K x+L x+M w=0 .
$$

The appearing vector $x(t) \in \mathbb{R}^{n}$ describes the set of state variables and the vector $w(t) \in \mathbb{R}^{m+p}$ describes the set of external variables.

In the sequel we will assume that (4) describes the plant which we will assume to be controllable and observable (see $[8,9]$ for definitions). We will assume that the compensator is a general memoryless linear system having input number $p$ and output number $m$. In other words the compensator is described through some static relations of the form

$$
\tilde{M} w=0,
$$

where $\tilde{M}$ is a constant matrix of size $m \times(m+$ $p$ ). The closed loop behavior is then described through

$$
\left[\begin{array}{cc}
\frac{d}{d t} K+L & M \\
0 & \tilde{M}
\end{array}\right]\left[\begin{array}{c}
x \\
w
\end{array}\right]=0
$$

For ease of presentation we prefer in the following to describe the compensator $\tilde{M} w=0$ through an image representation. For this let $F$ be a $(m+p) \times p$ constant matrix of rank $p$. If $\tilde{M}$ has rank $m$ and if $\tilde{M} F=0$ then (5) is equally well described through the image representation

$$
w=F \ell,
$$

where $\ell$ is a latent variable. The combined behavior of the interconnected system is then described through

$$
\left[\frac{d}{d t} K+L \mid M F\right]\left[\begin{array}{l}
x \\
\ell
\end{array}\right]=0
$$


Finally we define the unnormalized closed-loop characteristic polynomial by

$$
\phi_{F}(s):=\operatorname{det}[s K+L \mid M F] \text {. }
$$

The presentation we described so far reduces to the classical description in the following way: After a possible permutation of the coordinates of $w$ and after some possible row operations on the system of equations (4) it is always possible transform the pencil $[s K+L \mid M]$ into the familiar pencil

$$
\left[\begin{array}{ccc}
s I-A & 0 & -B \\
C & -I_{p} & D
\end{array}\right] .
$$

We would like to stress that (4) only reduced to a classical input/output description after a particular choice of input and output variables among the variables $w$. If $w$ was a priori partitioned into input variables $u$ and output variables $y, w=\left[\begin{array}{l}y \\ u\end{array}\right]$, then the class of systems representable by $(4)$ is strictly larger than the class of systems represented by

$$
\frac{d}{d t} x=A x+B u, \quad y=C x+D u .
$$

In the sequel it will be necessary to clarify what we understand under a generic set of linear systems having input number $m$, output number $p$ and McMillan degree $n$. For this recall that a subset $S$ of a vector space $\mathbb{R}^{k}$ is called a generic set if its complement $\mathbb{R}^{k} \backslash S$ is contained in the zero set of some nonzero polynomial in $x_{1}, \ldots, x_{k}$. Based on this we will identify a triple $(K, L, M)$ of matrices with a point in a vector space and we say a set of generalized first order systems forms a generic set whenever the corresponding set of matrix triples representing those systems forms a generic set. (Compare with [7, Definition 2.2]).

The main theorem which we will prove in this short note is now as follows. Identify the set of real polynomials of degree at most $n$ with the vector space $\mathbb{R}^{n+1}$ and identify the set of generalized compensators of the form (7) with the vector space $\mathbb{R}^{(m+p) p}$. Then we have:
Theorem 2 If $n<m p$ then the generalized pole placement map

$$
\begin{aligned}
\chi: \quad \mathbb{R}^{(m+p) p} & \longrightarrow \mathbb{R}^{n+1} \\
F & \longmapsto \operatorname{det}[s K+L \mid M F]
\end{aligned}
$$

is surjective for a generic set of real matrices $(K, L, M)$ of sizes $(n+p) \times n,(n+p) \times n$, and $(n+p) \times(m+p)$ respectively.

\section{Outline of Proof}

In this section we will give the proof Theorem 2. Our proof follows ideas developed by the authors in $[2,7]$ for the classical output feedback problem both for static ([2]) and dynamic ([7]) feedback compensators. Actually Theorem 2 is a corollary of [7, Theorem 3.4] although the following proof is more direct and less involved. For ease of readability we divide the proof in $3 \mathrm{sub}$ sections.

\subsection{A Sufficiency Condition}

We establish a sufficient criterion (Theorem 5) which guarantees that the pole placement map $\chi$ is surjective over the reals. The criterion is based on a linearization around a dependent compensator, an idea which appeared first in purely geometric language in [1] and was later worked out in more elementary terms independently by Leventides [3, 4], Wang [5] and Ariki [6].

Definition 3 A compensator $F$ will be called a dependent compensator for the system $(K, L, M)$ if $\phi_{F}(s)=0$.

The following properties of $\chi$ are immediate from the definition:

(i) $\chi(F S)=\chi(F)$ for any matrix $S \in \mathbb{R}^{p \times p}$ that has determinant 1 ;

(ii) $\chi(\lambda F)=\lambda^{p} \chi(F)$ for $\lambda \in \mathbb{R}$, i.e. $\chi$ is homogeneous of degree $p$.

Since $\chi$ is a polynomial map we may compute the Jacobian of $\chi$, denoted by $d \chi_{F}$, at each point of $F \in \mathbb{R}^{(p+m) p}$. The Jacobian is a linear transformation from $\mathbb{R}^{(m+p) p}$ to $\mathbb{R}^{n+1}$. By the standard multivariable Taylor series expansion one has

$$
\chi(F+\varepsilon H)=\chi(F)+\varepsilon d \chi_{F}(H)+
$$
terms of higher order in $\varepsilon$. 
As a consequence of property (i) above, the rank of the Jacobian can be at most

$$
(p+m) p-\left(p^{2}-1\right)=m p+1 .
$$

Compensators for which the Jacobian $d \chi_{F}$ is surjective will be important in the sequel. Following the language in [2] we introduce the following terminology:

Definition 4 The compensator $F$ will be called full if the Jacobian $d \chi_{F}$ is surjective.

Theorem 5 If a dependent full compensator exists for a system $(K, L, M)$, then the pole placement map $\chi$ is surjective.

Proof: Let $F$ be dependent and full compensator. By the inverse function theorem, an open neighborhood of $F$ is mapped onto an open neighborhood, say $\mathrm{V}$, of $\chi(F)=0$. Because any polynomial $\phi$ of degree at most $n$ may be written as $\phi=c \phi_{0}$ with $\phi_{0} \in V$ for some (sufficiently large) $c \in \mathbb{R}$, and since $\chi(\lambda F)=\lambda^{p} \chi(F)$ for any $\lambda \in \mathbb{R}$, this is already enough to prove that $\chi$ is surjective.

To the extent that the inverse function theorem is constructive, the proof provides also a way to compute a compensator that will assign a given set of poles. For details see $[7,5]$.

We conclude this subsection with an explicit formula of the Jacobian $d \chi_{F}$. For this recall that the adjoint of a $p \times p$ matrix $A$, denoted by adj $A$, is the $p \times p$ matrix defined by

$$
(\operatorname{adj} A)_{i j}:=\left((-1)^{i+j} A_{j i}\right)_{i, j=1}^{p}
$$

where $A_{j i}$ denotes the determinant of the ( $p-$ 1) $\times(p-1)$ matrix obtained from $A$ by removing the $j$-th row and the $i$-th column. Finally let tr denote the trace of a matrix.

\section{Lemma 6 The Jacobian}

$$
d \chi_{F}: \mathbb{R}^{(m+p) p} \longrightarrow \mathbb{R}^{n+1}
$$

is given by

$$
H \longmapsto \operatorname{tr}(\operatorname{adj}[s K+L \mid M F])[0 \mid M H],
$$

where $H$ is an arbitrary $(m+p) \times p$ constant matrix.
Corollary 7 Let $m_{j}, j=1, \ldots, m+p$ be the $j$ th column of $M$ and let $f_{i}(s), i=1, \ldots, n+p$ be the $i$-th row of adj $[s K+L \mid M F]$. Then the Jacobian $d \chi_{F}$ is full if and only if the polynomials $\left\{f_{i}(s) m_{j} \mid i=n+1, \ldots, n+p, j=1, \ldots, m+p\right\}$ span all polynomials of degree at most $n$.

\subsection{A Compensator Construction}

If $n<m p$ we will construct in this subsection for every system $(K, L, M)$ a particular dependent compensator.

Lemma 8 Assume $x(s) \in \mathbb{R}^{n}[s]$ and $w(s) \in$ $\mathbb{R}^{m+p}[s]$ are polynomial vectors of degree at most $p-1$, i.e.

$$
\left[\begin{array}{c}
x(s) \\
w(s)
\end{array}\right]=\sum_{i=1}^{p-1}\left[\begin{array}{c}
x_{i} \\
w_{i}
\end{array}\right] s^{i} .
$$

If

$$
[s K+L \mid M]\left[\begin{array}{l}
x(s) \\
w(s)
\end{array}\right]=0
$$

then the $(m+p) \times p$ matrix

$$
F:=\left[w_{0} \cdots w_{p-1}\right]
$$

describes a dependent compensator.

Proof: Let $X:=\left[x_{0} \cdots x_{p-1}\right]$. Then

$$
\begin{aligned}
0 & =\operatorname{det}\left([s K+L \mid M]\left[\begin{array}{cc}
I_{n} & X \\
0 & F
\end{array}\right]\right) \\
& =\operatorname{det}\left([s K+L \mid M]\left[\begin{array}{cc}
I_{n} & 0 \\
0 & F
\end{array}\right]\right) \\
& =\operatorname{det}[s K+L \mid M F] .
\end{aligned}
$$

Lemma 9 If $n<m p$ then there exists nontrivial vectors $x(s) \in \mathbb{R}^{n}[s]$ and $w(s) \in \mathbb{R}^{m+p}[s]$ satisfying (14).

Proof: Let $x_{i}, w_{i}$ be defined as in (13). Let

$$
R:=\underbrace{\left[\begin{array}{cccc|ccccc}
L & 0 & \ldots & 0 & M & 0 & \ldots & \ldots & 0 \\
K & L & \ddots & \vdots & 0 & M & \ddots & & \vdots \\
0 & K & \ddots & 0 & \vdots & \ddots & \ddots & \ddots & \vdots \\
\vdots & \ddots & \ddots & L & \vdots & & \ddots & M & 0 \\
0 & \ldots & 0 & K & 0 & \ldots & \ldots & 0 & M
\end{array}\right]}_{2 p-1 \text { blocks }} .
$$


Then (14) is equivalent with

$$
R\left[\begin{array}{c}
x_{0} \\
\vdots \\
x_{p-1} \\
w_{0} \\
\vdots \\
w_{p-1}
\end{array}\right]=0 .
$$

Since $R$ has $p(n+p)$ rows and $(p-1) n+p(m+p)$ columns (16) has a nontrivial solution as soon as $n<m p$.

\subsection{Genericity}

In the last subsection we described a procedure which led from a system $(K, L, M)$ to dependent compensator $F$. Unless the matrix $R$ has corank 1 the compensator $F$ was not unique.

In the following we will adapt this procedure resulting in a polynomial function which assigns to a triple $(K, L, M)$ a unique dependent compensator.

At the end of the subsection we then show that for a generic set of systems this polynomial map results in a dependent and full compensator. This will complete then the proof.

Assume now that $n<m p$. For simplicity we also will assume that $m p-n \leq p$ and leave the technical difficulties for the other (seemingly easier) cases to the reader.

Extend the matrix $R$ to a square matrix

$$
Q:=\left[\begin{array}{ll} 
& R \\
0 & I_{m p-n}
\end{array}\right]
$$

and let the vector

$$
v:=\left(x_{0}^{t}, \ldots, x_{p-1}^{t}, w_{0}^{t}, \ldots, w_{p-1}^{t}\right)^{t}
$$

be the rightmost column vector of $\operatorname{adj} Q$. Note that by definition $v$ is in the kernel of $R$ and the components of $v$ are polynomial functions of the system parameters.

In this way we associated in a polynomial way to every triple $(K, L, M)$ a dependent compensator. By Corollary 7 it follows that the set of triples

$$
(K, L, M) \in \mathbb{R}^{(n+p) \times(2 n+m+p)}
$$

whose associated dependent compensator is not full is a set determined by polynomial equations.
This still does not prove genericity of arbitrary pole placement however, since it might happen that the polynomial equations turn out to be trivial (of the form $0=0$ ), in which case $d \chi_{F}$ would be rank deficient for all triples $(K, L, M)$. To show that this situation does not occur, the last step in the proof therefore is to come up with one triple $(K, L, M)$ for which the Jacobian $d \chi_{F}$ does indeed have full row rank.

In [2] a similar construction for a dependent compensator was given in the situation where the plants consisted of strictly proper transfer functions only. Let $(A, B, C)$ be the example provided in [2]. Let

$$
K:=\left[\begin{array}{l}
I \\
0
\end{array}\right], L:=\left[\begin{array}{c}
-A \\
C
\end{array}\right], M:=\left[\begin{array}{cc}
0 & -B \\
-I & D
\end{array}\right] .
$$

One readily verifies that the polynomial construction described in this paper and applied to this particular triple $(K, L, M)$ results in a dependent compensator which is column equivalent to the polynomial dependent compensator computed in [2] for the triple $(A, B, C)$. This completes the proof.

\section{Some Geometric Remarks}

A representation of the form (4) describes a behavior $\mathcal{B}(K, L, M)$ in the sense of Willems [9]. Note that a change of basis in the state variables and elementary row operations have no effect on the behavior, i.e. if $T \in G l_{n+p}$ and $S \in G l_{n}$ then

$$
\mathcal{B}(K, L, M)=\mathcal{B}\left(T K S^{-1}, T L S^{-1}, T M\right) .
$$

On the other hand if $(K, L, M)$ and $(\tilde{K}, \tilde{L}, \tilde{M})$ are two minimal triples representing the same controllable external behavior then it is known that

$$
(\tilde{K}, \tilde{L}, \tilde{M})=\left(T K S^{-1}, T L S^{-1}, T M\right)
$$

for some invertible matrices $T \in G l_{n+p}$ and $S \in$ $G l_{n}$. Consequently certain equivalence classes under the group action $G l_{n+p} \times G l_{n}$ represent uniquely certain behaviors.

Let $Y \subset \mathbb{R}^{(n+p) \times(2 n+m+p)}$ be the set of all triples $(K, L, M)$ which are controllable, i.e. $\operatorname{rank}[s K+L \mid M]=n+p$ for all $s \in \mathbb{C} \cup\{\infty\}$ and 
which satisfy the property that $\operatorname{rank}[s K+L]=n$. The main result in [10] states that the geometric quotient

$$
\mathcal{H}_{m, p}^{n}:=Y /\left(G l_{n+p} \times G l_{n}\right)
$$

has the structure of a smooth projective variety, i.e. it has also the structure of a compact manifold. The space $\mathcal{H}_{m, p}^{n}$ also is studied in the algebraic geometry literature and it is often referred to as a Quot scheme. It follows from the previous remarks that $\mathcal{H}_{m, p}^{n}$ parameterizes the set of linear time invariant behaviors having a fixed McMillan degree $n$, a fixed input number $m$ and a fixed output number $p$. Moreover $\mathcal{H}_{m, p}^{n}$ contains the variety of proper transfer functions as a Zariski open and therefore dense subset.

As a consequence of Theorem 2 and the results reported in [10] we therefore have:

Theorem 10 If $n<m p$ then the generalized pole placement map $\chi$ is surjective for a generic subset of the variety $\mathcal{H}_{m, p}^{n}$.

\section{Conclusion}

In this note we studied the pole placement problem for a generalized first order system using generalized first order compensators. Using the method of linearization around a dependent compensator we established the result that arbitrary pole placement is possible for the generic plant as soon as the McMillan degree $n$ of the plant is strictly less than the input number times the output number of the plant.

\section{References}

[1] X. Wang, "Pole placement by static output feedback", Journal of Math. Systems, Estimation, and Control, vol. 2, no. 2, pp. 205-218, 1992.

[2] J. Rosenthal, J. M. Schumacher, and J. C. Willems, "Generic eigenvalue assignment by memoryless real output feedback", CWI Report BS-R9438, Dec. 1994, To appear in Systems \& Control Letters.

[3] J. Leventides, Algebrogeometric and Topological Methods in Control Theory, $\mathrm{PhD}$ thesis, City University London, Sept. 1993.
[4] J. Leventides and N. Karcanias, "Global asymptotic linearisation of the pole placement map: A closed form solution for the constant output feedback problem", Tech. Rep., City University London, Sept. 1994, To appear in Automatica.

[5] X. Wang, "Grassmannian, central projection and output feedback pole assignment of linear systems", Preprint, to appear in IEEE Trans. Automat. Control., July 1994.

[6] S. Ariki, "Generic pole assignment via dynamic feedback", Preprint, Oct. 1994.

[7] J. Rosenthal and X. Wang, "Output feedback pole placement with dynamic compensators", CWI Report BS-R9516, May 1995.

[8] M. Kuijper, First-Order Representations of Linear Systems, Birkhäuser, Boston, 1994.

[9] J. C. Willems, "Paradigms and puzzles in the theory of dynamical systems", IEEE Trans. Automat. Control, vol. AC-36, no. 3, pp. 259-294, 1991.

[10] M. S. Ravi and J. Rosenthal, "A general realization theory for higher order linear differential equations", Systems \& Control Letters, vol. 25, no. 5, pp. 351-360, 1995.

[11] C. I. Byrnes, "Pole assignment by output feedback", in Three Decades of Mathematical System Theory, H. Nijmeijer and J. M. Schumacher, Eds., Lecture Notes in Control and Information Sciences \# 135, pp. 31-78. Springer Verlag, 1989.

[12] M. Kuijper and J. M. Schumacher, "Realization of autoregressive equations in pencil and descriptor form", SIAM J. Control Optim., vol. 28 , no. 5 , pp. 1162-1189, 1990.

[13] J. C. Willems, "On interconnections, control and feedback", Preprint, submitted to IEEE AC, July 1994. 